\title{
Radiología pediátrica en Atención Primaria
}

\author{
H. Cortina Orts \\ Servicio de Radiodiagnóstico. Hospital Infantil La Fe. Valencia. España.
}

\section{Resumen}

Revisión y puesta al día de claves de imagen decisivas, según el autor, en patología pulmonar, gastroenterología y sistema musculoesquelético, especialmente significativas por su frecuencia y actualidad. infantil.

Palabras clave: Neumonía. Tuberculosis pulmonar. Reflujo gastroesofágico. Maltrato

Pediatric radiology in Primary Care

Abstract

Revision and update of decisive imaging keys in pulmonary, gastroenterological and musculoskeletal conditions, especially significative for their frequency and present interest.

Key words: Pneumonia. Pulmonary tuberculosis. Gastroesophageal reflux. Child abuse.

\section{Introducción}

Durante los últimos años hay un clima de especial sensibilización en el entorno de la Pediatría a partir de la información, ahora conocida, acerca de los efectos cancerígenos evolutivos a raíz de la explosión de la bomba atómica. Sesenta años después se conocen las cifras directas de pacientes que, entonces en edad infantil, han alcanzado ahora edades propicias para la aparición de los diferentes tipos de cáncer. Hay mayor incidencia de cáncer, estadísticamente significativa, en la población irradiada en el rango de los 50 mSv, es decir, en el espectro de la radiología convencional y la tomografía computarizada (TC) ${ }^{1}$. Esta incidencia se incrementa por debajo de los diez años y progresa conforme se aproxima al periodo neonatal, relacionada con el mayor potencial de vida y la alta capacidad mitótica en estos años iniciales².

Héctor Cortina Orts: cortina_hec@hotmail.com

El autor declara no presentar conflictos de intereses en relación con la preparación y publicación de este artículo. 
Radiación ambiente e irradiación en Radiología: equivalencias

Las radiaciones ionizantes son responsables de la radiación de fondo que recibe la población. La dosis media anual es de 2,4 mSv.

En la Directiva 1997-743/Euratom del Consejo de la Unión Europea de $30 / 6 / 97^{2}$, se establece como orientación la "Guía de indicaciones para la correcta solicitud de pruebas de diagnóstico por imagen"3,4, que incluye el cuadro de equivalencias con respecto a la radiación ambiente, tomando como unidad la radiografía de tórax (dosis $<0,1 \mathrm{mSv}$ ). Las equivalencias significativas se muestran en la tabla 1.

\section{Un problema de Salud Pública}

Estos datos no justifican posturas radicales. El riesgo adicional de cáncer por la práctica de una radiografía de tórax es de 1/1 000000 . Y el de una TC de abdomen, por ejemplo, supone aproximada- mente un incremento de 1/2000. Para un individuo concreto, considerando que el riesgo de la población general de padecer cáncer es de un 30\%, el incremento del riesgo por estudios radiológicos es escasísimamente significativo. Por ello, se entiende que el riesgo individual ante una exploración tanto convencional como de TC es muy bajo, de forma que, para un caso concreto, si la exploración está indicada, el balance riesgo-beneficio favorece abrumadoramente al beneficio.

Pero cuando este factor se multiplica por el formidable número de estudios anuales practicados a la población se convierte en estadísticamente significativo. Por ello, hoy se admite que la irradiación diagnóstica con fines médicos supone un riesgo de Salud Pública 5 .

Las normas deben ir orientadas a vigilar e incrementar los sistemas de protección de pacientes en los servicios de Radiodiagnóstico y a eliminar toda exploración innecesaria.

Tabla 1. Procedimiento diagnóstico. Radiografias de Tórax. Equivalencia con respecto a la radiación ambiente

\begin{tabular}{l|l|r|r}
\hline Radiografía & Tórax & 1 & 1,5 días \\
\cline { 2 - 4 } & Cráneo & 3,5 & 11 días \\
\cline { 2 - 4 } & Abdomen & 50 & 6 meses \\
\cline { 2 - 4 } & Estudio digestivo & 150 & 16 meses \\
\cline { 2 - 4 } & Enema opaco & 350 & 3 años \\
\hline Tomografía computarizada & Cráneo & 115 & 1 año \\
\hline \multirow{2}{*}{} & Tórax & 400 & 3,6 años \\
\cline { 2 - 4 } & Abdomen & 500 & 4,5 años \\
\hline
\end{tabular}


Estudios en patología torácica

La radiografía simple de tórax es, en más del $90 \%$ de casos, principio y fin de estudio en patología tanto aguda como crónica. La proyección anteroposterior es, de entrada, suficiente para un diagnóstico correcto ${ }^{6,7}$. Supone una reducción de dosis de un $60 \%$.

\section{Neumonía bacteriana}

Prácticamente desaparecido el Haemophilus influenzae y pérdida la agresividad del Staphilococcus aureus, el Streptococcus pneumoniae-11 es el agente etiológico de neumonía más frecuente en la edad pediátrica. Durante los últimos 20 años algunas cepas han experimentado una resistencia progresiva con aparición de serotipos de alta agresividad y capacidad necrotizante.

- Radiografía convencional. El patrón inicial es el alveolar, propio de cualquier afectación bacteriana. Las complicaciones como el derrame pleural o la falta de respuesta a la antibioterapia adviertan de su severidad (figura 1).

- Ecografía:

- Caracterización del derrame pleural. Diferencia el derrame libre de

Figura 1. Ecografía de parénquima pulmonar en paciente con neumonía; área hipoecoica por isquemia compatible con neumonía necrotizante. 
las formas complicadas que comprenden desde la presencia de finos filamentos de fibrina hasta la formación de múltiples tractos fibróticos. La ecografía muestra una calidad de información superior a la TC.

- Caracterización de la consolidación parenquimatosa pulmonar. Identifica la presencia de áreas de baja ecogenicidad, características de neumonía necrotizante.

\section{Neumonía por Mycoplasma pneumoniae y virus}

Las lesiones provocadas por el Mycoplasma pneumoniae ${ }^{12}$ y los virus afectan directamente al epitelio ciliado de la tráquea a los bronquiolos terminales. Los hallazgos están relacionados con el menor tamaño de la luz bronquial, el escaso desarrollo inicial de los sistemas colaterales de ventilación y la mayor capacidad de secreción mucosa del pulmón pediátrico. El resultado es la preponderancia de la hiperinsuflación pulmonar valvular en la bronquiolitis del lactante. Más adelante, los patrones tanto de origen viral como por Mycoplasma pneumoniae muestran predominio de afectación reticular secundaria al edema bronquial y perivascular con áreas de enfisema o ate- lectasia por tapones mucosos y discretas áreas de alveolización.

\section{Tuberculosis pulmonar} en fase inicial: infección frente a enfermedad tuberculosa

El acúmulo de bacterias en las partículas infectadas genera un foco pulmonar, el chancro de Gohn. Es la primoinfección tuberculosa. Si los bacilos sobreviven son drenados por vía linfática a los ganglios regionales. El conjunto de chancro, linfangitis y adenopatías, el complejo de Ranke, significa enfermedad tuberculosa.

Se acepta que para el tratamiento de la primoinfección tuberculosa es suficiente la administración de un solo fármaco. La enfermedad tuberculosa precisa al menos de tres fármacos; es fundamental, por tanto, la diferenciación entre ambas fases.

El diagnóstico de enfermedad tuberculosa se sustenta en: constatación de entorno bacilífero y prueba de tuberculina positiva, que significan infección, y detección por imagen de adenopatías mediastínicas, que traduce extensión y, por tanto, enfermedad. Por ello, la radiología es crucial. De entrada, en un determinado número de pacientes, sobre todo en los casos Índice, la radiografía muestra las adenopatías mediastínicas (la proyec- 
ción lateral es extraordinariamente útil para la identificación de las adenopatías mediastínicas subcarinales, muy frecuentes). El estudio radiológico ha finalizado.

Pero si la radiografía simple es negativa o dudosa, la TC muestra extraordinaria sensibilidad para la detección y caracterización de las adenopatías ${ }^{13-16}$ (hasta un 60\%). No obstante, hay una controversia reciente basada en el predominio, en algunas series, de captación homogénea tras la administración de contraste, lo que indicaría ausencia de caseificación y un carácter únicamente reactivo, más próximo a la pri- moinfección ${ }^{17,18}$. No es, sin embargo, nuestra experiencia (figura 2 ).

\section{Pulmón hiperclaro más allá del cuerpo extraño}

Sospecha de anillo vascular. Los anillos vasculares más frecuentes son: arteria pulmonar izquierda aberrante y doble arco aórtico. Un esofagograma normal descarta anillo vascular.

\section{Neumotórax localizado por fenómeno de vacío}

Contiguo a la atelectasia aguda de un lóbulo pulmonar, especialmente de los

Figura 2. Paciente de cuatro años de edad, con febrícula de 15 días. Tuberculina positiva. Radiografía de tórax normal. Adenopatías hiliares y subcarinales con captación periférica tras la administración de contraste indicativa de abscesificación central.

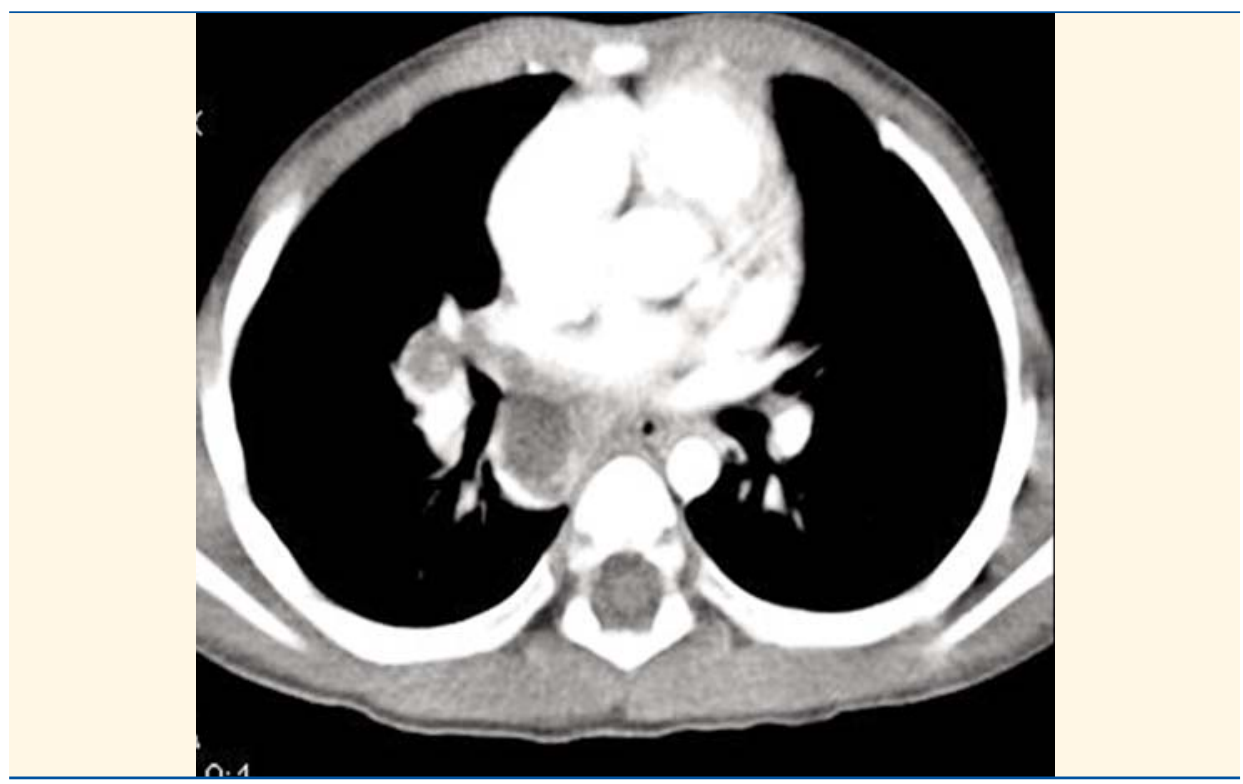


superiores, sobre todo derecho, por oclusión del bronquio correspondiente por cuerpo extraño o tapón mucoso en contexto de bronquiolitis, asma o proceso infeccioso (figura 3). Descrito por Berdon $^{19}$, se sugiere que este gas no es aire, sino mezcla de componentes, predominantemente nitrógeno, procedentes de estructuras vasculares vecinas.

\section{Atelectasia crónica}

La atelectasia de larga duración obliga a contemplar la posibilidad de tumor intrabronquial a identificar mediante TC. El más frecuente es el tumor carcinoide $(90 \%)^{20}$.

\section{Patología abdominal}

La indicación de la radiografía simple de abdomen es muy limitada. En el 95\% de los casos supone una irradiación gratuita del paciente. Hay que recordar que equivale a 50 radiografías de tórax.

La radiografía simple no está indicada en: dolor abdominal inespecífico, preferentemente periumbilical; dolor abdominal recurrente $e^{21} ; y$ retención fecal.

\section{Indicaciones de la radiografía} simple

- Sospecha de obstrucción intestinal. Habitualmente se observa un patrón aéreo obstructivo característico. Sin embargo, la ausencia de neumatización intestinal es relativamente frecuente. En un contexto clínico de dolor abdominal inespecífico no tiene valor patológico. Pero cuando el cuadro clínico sugiere obstrucción intestinal, la falta de neumatización obliga al estudio ecográfico abdominal para identificar la repleción líquida de asas intestinales y el hiperperistaltismo característico del íleo mecánico. E incluso, se puede detectar el nivel de la obstrucción.

- Sospecha de cólico nefrítico. Composición de los cálculos renales en la edad pediátrica: oxalato cálcico 75\%; carbonato de apatita 15\%; cistina, ácido úrico $2 \%$. Puesto que el $90 \%$ son radioopacos, son visibles con radiografía simple.

\section{Estudios digestivos}

Tránsito intestinal: no indicado, de entrada, en el reflujo gastroesofágico del lactante. Recordar que equivale a 150 radiografías de tórax ${ }^{22,23}$.

\section{Patología del sistema musculoesquelético}

Claves diferenciales con respecto al adulto: elasticidad, capacidad de remodelación y presencia del cartílago de crecimiento. 
Figura 3. Paciente de dos años de edad, con tos y fiebre alta desde hace 48 horas. A. Atelectasia completa de lóbulo superior izquierdo con pseudoneumotórax contiguo. B. Seis horas después tras aspiración de tapones mucosos: pequeñas atelectasias residuales.

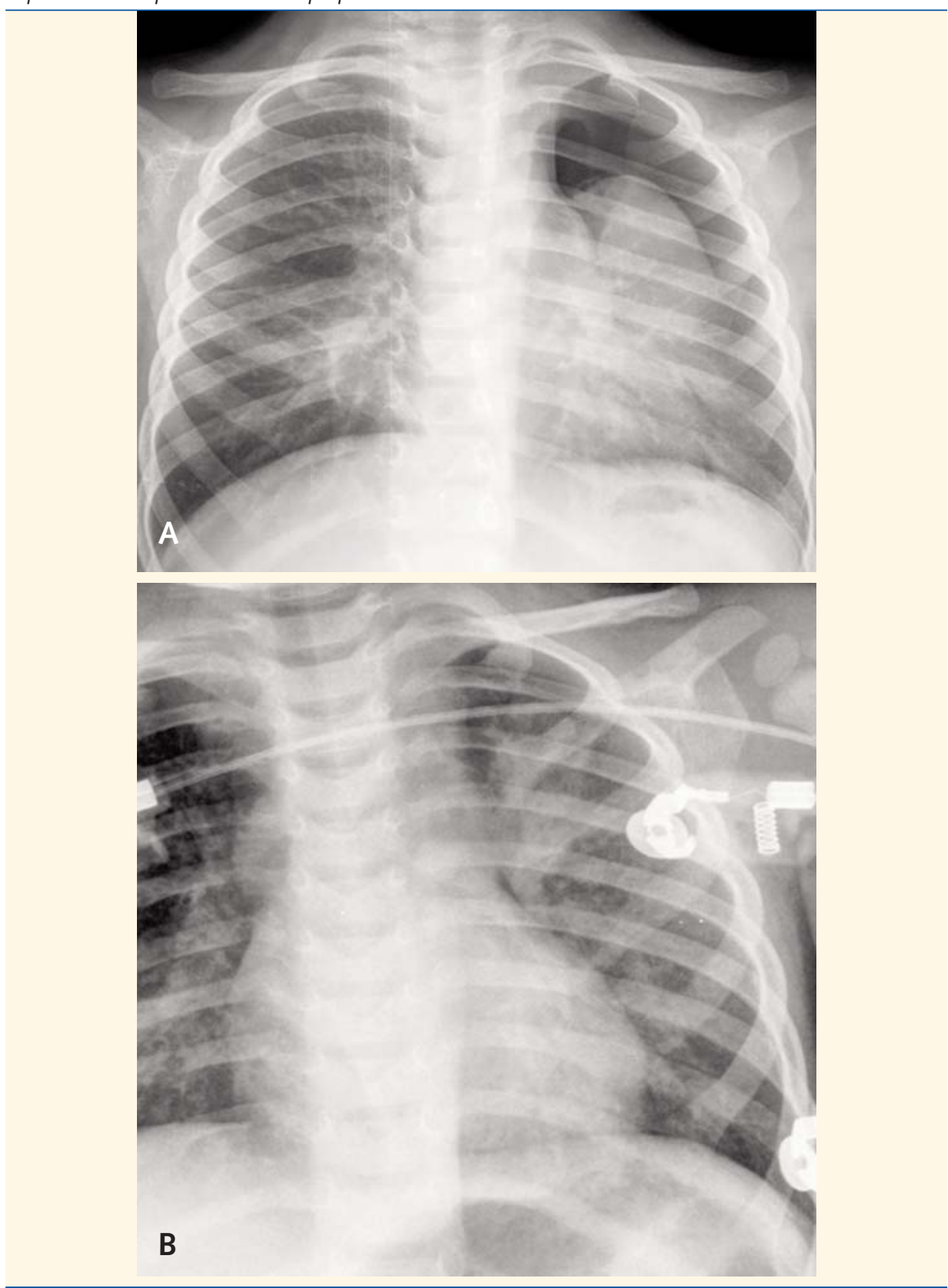




\section{Fracturas Toddler}

Fractura del niño que empieza a caminar. El apoyo inestable provoca, en algún momento, una torsión del miembro inferior sobre sí mismo, que se traduce en una fractura espiroidea, casi siempre de tibia. Una fractura Toddler previa a la marcha es altamente sugestiva de síndrome de maltrato infantil.

\section{Síndrome de maltrato infantil}

No es patología de hospital de referencia: el tratamiento de las fracturas no tiene especial complejidad, y las secuelas radiológicas llegan a cualquier con- sulta externa o centro de Atención Primaria.

- Fracturas en asa de cubo y en esquina. Patognomónicas de maltrato infantil. Las fracturas son inmediatas al cartílago fisario, quedando una fina banda ósea adherida que, en función del ángulo del rayo incidente, originan estas imágenes ${ }^{24,25}$ (figura 4).

- Fracturas costales. Una fractura costal por debajo del año de vida sugiere maltrato. Las fracturas de arcada posterior, especialmente las múltiples, indican maltrato ${ }^{26}$.

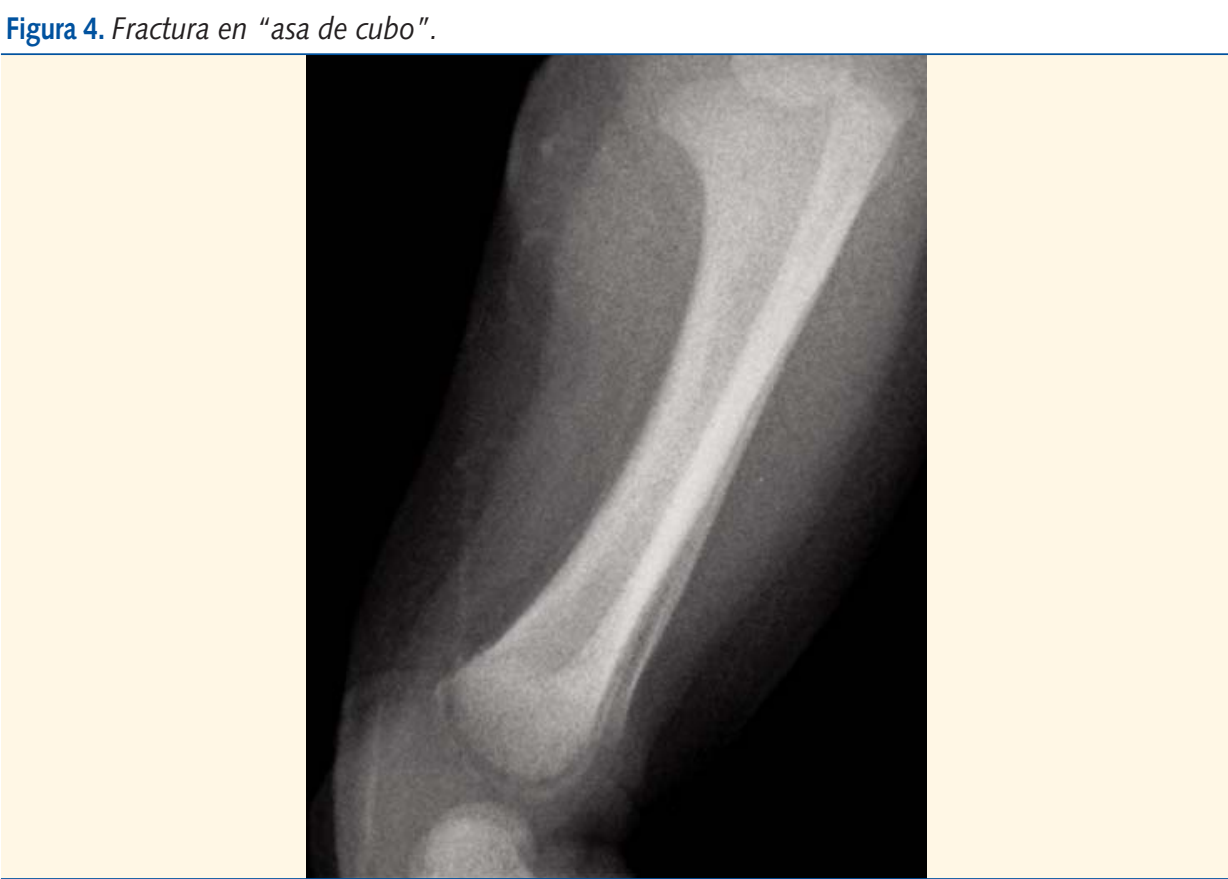




\section{Coxalgia aguda no traumática}

- Sin afectación de estado general, analítica negativa. Un alto porcentaje corresponde a sinovitis. No procede estudio radiográfico. Fase aguda: ecografía; persistencia de sintomatología pasados 15 días: radiografía y, en función de clínica y hallazgos, resonancia magnética (RM).

- Con afectación de estado general, si la analítica es positiva, realizar una ecografía (¿artritis séptica?); si es negativa, realizar una RM (¿osteomielitis aguda?, ¿piomiositis?).

\section{Cadera en abducción}

Forma parte de las deformidades prenatales de moldeamiento. Claves: asimetría de pliegues; posición espontánea en abducción de un miembro; acortamiento (aparente) del miembro contralateral; $y$, al nacimiento, maniobras de Barlow y Ortolani negativas.

El origen es una posición anómala intrauterina, en abducción, de un miembro. Al nacimiento, el hallazgo queda enmascarado por la postura espontánea de los miembros en flexión. Las maniobras de Ortolani y Barlow son negativas porque las caderas son nor-

Figura 5. Cadera izquierda en abducción. Aparente dismetría y asimetría de pliegues. 
males. Cuando, más adelante, el lactante estira las piernas, se descubre en espontáneo la posición viciada en abducción de un miembro por contractura de su musculatura abductora. Cuando durante la exploración física en decúbito prono el pediatra pretende la rectificación de la postura anómala provoca un disbalance de pelvis que condiciona el aparente acortamiento del miembro contralateral y la asimetría de pliegues (figura 5). Patología de gran prevalencia. Habitualmente el pronóstico es excelente.

\section{Bibliografía}

1. Pierce DA, Shimizu Y. Studies of the mortality of atomic bomb survivors. Report 12, part I. Cancer 1950-1990. Radiat Res. 1996;146:1-127.

2. Directiva 1997/43 Euratom del Consejo de la Unión Europea de 30 de Junio de 1997, sobre la protección de la salud frente a los riesgos derivados de las radiaciones ionizantes en exposiciones médicas (DOL 180, de 09/07/1997).

3. Guía de indicaciones para la correcta solicitud de pruebas de diagnóstico por imagen. Comisión Europea. Dirección de Medio Ambiente. Oficina de Publicaciones oficiales de las Comunidades Europeas. Valencia: Edición Generalitat Valenciana. Consellería de Sanidad; 2001.

4. Brenner DJ, Elliston CD. Estimated risk of radiation-induced fatal cancer from Pediatric CT. AJR. 2001;176:289-96.

5. Leung DP, Dixon AK. Clinicoradiological meetings: are they worthwhile? Clin Radiol. 2002;46:279-80.

6. Rigsby CK. Is the radiography sufficient to evaluate for pneumonia in children? Pediatr Radiol. 2004;27:315-19.

7. Kotagul UL. Does the lateral chest radiograph affect treatment? Radiology. 1997;15: 116-20.
8. Donelly LF. Maximizing the usefulness of imaging in children with community-adquirid pneumonia. AJR. 1999;172:505-12.

9. Asensio O, Blanco J, Moreno A, Pérez J, Salcedo A, Sanz $L$ et al. Tratamiento de los derrames pleurales paraneumónicos. An Esp Pediatr. 2001;54:272-82.

10. Yang PC, Luh KT, Chang DB, Wu HD, Yu $\mathrm{CJ}$, Kuo SH et al. Value of sonography in determining the nature of pleural effusion: analysis of 320 cases. AJR. 1992;159:29-33.

11. Calder $A$, Owens $C M$. Imaging of parapneumonic pleural effusions and empyema in children. Pediatr Radiol. 2009;39:527-37.

12. Ramanathan JS, Swischuk LE. Spectrum of clinical and radiographic findings in pediatric Mycoplasma Pneumoniae. RadioGraphics. 2001; 21:121-31.

13. Delacourt C, Mani TM. Computed Tomography with normal chest radiograph in tuberculous infection. Arch Dis Child. 1993;69: 430-2.

14. Andronikou S, Wieselthaler N. Modern imaging of tuberculosis in children: thoracic central nervous system and abdominal tuberculosis. Pediatr Radiol. 2004;34:861-75.

15. Kim WS, Moon WK, Kim IO, Lee HJ, Im JG, Yeon KM et al. Pulmonary tuberculosis in 
children: evaluation with CT. AJR. 1997;168(4): 1005-9.

16. Andronikou S, Joseph E. CT scanning for the detection of tuberculous mediastinal and hilar lymphadenopathy in children. Pediatr Radiol. 2004;34:232-6.

17. Delacourt D, Albertini M, Decludt B. ¿Quels son les examens utiles davant un enfant exposé, assymptomatic ayant une intra-dermorreaction à la tuberculine positive et une radiographie thoracique normale? Rev Mal Respir. 2004;21:513-23.

18. Gómez Pastrana D, Carceller Blanchard A. ¿Debe realizarse una tomografía computarizada torácica a los niños con infección tuberculosa sin enfermedad aparente? An Pediatr (Barc). 2007; 67:585-93.

19. Berdon WE, Dee GJ. Localizad Pneumothorax Adjacent to a collapsed lobe: A Sign of Bronchial Obstrution. Radiology. 1984;150:691-4.
20. Encinas JL, Ávila JF. Tumor carcinoide bronquial y apendicular. An Pediatr (Barc). 2006; 64:474-7.

21. Urruzuno $P$, Tellería C. Dolor abdominal. An Esp Pediatr. 2002;56:452-8.

22. Izquierdo $M$, Marco A. Controversias sobre el reflujo Gastroesofágico. Pediátrika. 2006; 22:42-7.

23. Salvatore $S$. The natural curse of Gastroesophageal Reflux. Acta Pediátr. 2004;30:355-7.

24. Kleinman PK, Marks SC. The methaphyseal lesion in abused infants: a radiologic-histopathologic study. AJR. 1986;146:895-905.

25. Kleinman PL, Kleinman PK. Suspected infant abuse: radiographic skeletal survey practices in pediatric health care facilities. Radiology. 2004;233:477-85.

26. Offiah A, Rick R. Skeletal imaging of child abuse (non-accidental injury). Pediatr Radiol. 2009;39:461-70. 